\title{
Methods for diagnosing perceived age on the basis of an ensemble of phenotypic features
}

This article was published in the following Dove Press journal:

Clinical, Cosmetic and Investigational Dermatology

17 April 2014

Number of times this article has been viewed

\author{
Mireia Coma' \\ Raquel Valls' \\ José Manuel Mas' \\ Albert Pujol' \\ Miquel Angel Herranz ${ }^{2}$ \\ Vicente Alonso 2,3 \\ Jordi Naval ${ }^{2}$
}

'Anaxomics Biotech, Barcelona, Spain; ${ }^{2}$ Genocosmetics Laboratory,

Barcelona, Spain; ${ }^{3}$ Unidad de

Dermatología del Hospital Nisa

9 Octubre de Valencia, Valencia, Spain

Correspondence: Jordi Naval

R \& D Department, Genocosmetics

Laboratory, Rambla Catalunya ${ }^{\circ} 81$

$8^{\circ}-3^{\circ}, 08008$ Barcelona, Spain

Tel +34936 II0648

Email rd-operations@genocosmetics.com
Background: Perceived age has been defined as the age that a person is visually estimated to be on the basis of physical appearance. In a society where a youthful appearance are an object of desire for consumers, and a source of commercial profit for cosmetic companies, this concept has a prominent role. In addition, perceived age is also an indicator of overall health status in elderly people, since old-looking people tend to show higher rates of morbidity and mortality. However, there is a lack of objective methods for quantifying perceived age.

Methods: In order to satisfy the need of objective approaches for estimating perceived age, a novel algorithm was created. The novel algorithm uses supervised mathematical learning techniques and error retropropagation for the creation of an artificial neural network able to learn biophysical and clinically assessed parameters of subjects. The algorithm provides a consistent estimation of an individual's perceived age, taking into account a defined set of facial skin phenotypic traits, such as wrinkles and roughness, number of wrinkles, depth of wrinkles, and pigmentation. A nonintervention, epidemiological cross-sectional study of cases and controls was conducted in 120 female volunteers for the diagnosis of perceived age using this novel algorithm. Data collection was performed by clinical assessment of an expert panel and biophysical assessment using the ANTERA $3 \mathrm{D}^{\circledR}$ device.

Results and discussion: Employing phenotype data as variables and expert assignments as objective data, the algorithm was found to correctly classify the samples with an accuracy of 92.04\%. Therefore, we have developed a method for determining the perceived age of a subject in a standardized, consistent manner. Further application of this algorithm is thus a promising approach for the testing and validation of cosmetic treatments and aesthetic surgery, and it also could be used as a screening method for general health status in the population.

Keywords: perceived age, algorithm, dermatology, diagnostic method, skin aging

\section{Introduction}

Physical appearance plays a central role in social interactions. "What is beautiful is good" wrote the Greek poet Sappho almost three millennia ago, and this affirmation still holds true in our society. Tangible proof of the prominent role of beauty in our society is the $\$ 220$ billion (worldwide annual US dollars) in revenues that was obtained in 2012 by the companies in the cosmetics sector. ${ }^{1}$

There is a tight connection between external appearance and age: young looks are associated with beauty, and that is why people try to keep hold of their youth. When dealing with aging and appearance, however, clear terminology is required. "Chronological age" is typically defined as a person's lifespan, that is, the amount of years that have passed since that person was born. Because tissues deteriorate at 
different rates depending on the individual, this concept fails to provide a clear indicator of the aging process. Therefore, the term "biological age" has been coined to describe the functional status of an individual in reference to others possessing the same chronological age. ${ }^{2}$

Among the collection of changes that accompany the aging process, the progressive deterioration of external appearance is probably the most notorious one. Accounting for this, the term "perceived age" comes into play, defined as the age that a person is visually estimated to be on the basis of physical appearance. In all cases, years and months thereof are used as standard measurement units.

A close link between biological and perceived age has been identified, because people who retain their functional capabilities usually show a younger appearance. So, perceived age is a good estimate of health in elderly populations, and hence it was recently shown to be a clinical marker for assessment of "healthy" aging. ${ }^{3}$ Subjects looking old for their age had a greater risk of both morbidity and mortality. Also, higher perceived age has been associated with high serum glucose levels, ${ }^{4}$ cortisol levels, ${ }^{5}$ and depression. ${ }^{6,7}$

More importantly, neither perceived age nor biological age are strictly dependent on chronological age, a fact that has profound implications for the cosmetic industry. Some people display an astonishingly young look in relation to their appearance, whereas others - sometimes, one of two twins - deteriorate much more rapidly. These differences can be a result of various intrinsic and extrinsic factors including, but not limited to, exposure to sunlight, pollution, nicotine, and diet or sleeping habits. ${ }^{8}$ Nongenetic factors have a great contribution to perceived age: changes in facial features, as skin wrinkling, ${ }^{3}$ skin color homogeneity, ${ }^{9,10}$ lip size, ${ }^{3}$ and sag ${ }^{11}$ have all been linked to perceived age.

The cosmetic industry uses perceived age assessment to determine the efficacy of treatments - for example, to quantify the efficacy of multisyringe hyaluronic acid treatment ${ }^{12}$ or plastic surgery. ${ }^{13}$ Perceived age is measured by clinical assessment, conventionally through photographic images. ${ }^{14,15}$ Still, as the Irish novelist Margaret Wolfe Hungerford immortalized in her classic Molly Bawn, "beauty is in the eye of the beholder": despite the use of accepted grading scales, ${ }^{16}$ estimation of age relies on subjective human assignments. In addition, hiring an entire team of experts is expensive and time consuming, and hence not feasible for large-scale assessments. Therefore, there is a need for an objective method to determine the perceived age of a person in a faster, cheaper, and standardized manner.
The diagnosis of a person's perceived age could be applied as an easy and noninvasive method to estimate a person's health in the same way that abdominal girth is a marker of metabolic syndrome ${ }^{17}$ or cardiovascular events, ${ }^{18}$ as well as to evaluate the efficacy of cosmetic treatments such as contact thermography, morphometric measures of thigh circumference, and microcirculation evaluation of cellulite. $^{19}$

In the present article, a new and standardized method for diagnosing perceived age is described and subsequently applied to a group of volunteers. This method consists of a mathematical algorithm that measures phenotypic features of individuals and classifies them to evaluate a person's skin aging. The measurement of a person's perceived age over time could be useful for the validation of treatments such as cosmetic treatment, aesthetic surgery, exercise, nutritional complements, diets, and alternative therapies including relaxation, laughter therapy, personal growth therapy, and nutritional complements.

\section{Methods}

We designed an artificial neural network (ANN) algorithm that provides an objective estimation of an individual's perceived age on the basis of phenotypic features. Thus, phenotypic data is the input value of the algorithm, whereas perceived age is the output. In order to test the validity of the method and check its accuracy, it was applied to a group of volunteers of ages ranging from 41 to 49 years old.

\section{Study design}

A nonintervention, epidemiological cross-sectional study of cases and controls was conducted in 120 female volunteers for the diagnosis of perceived age using the novel algorithm. The epidemiological study was conducted at the Hospital of Nisa, Valencia, Spain. The study received the approval of the Autonomic Ethics Committee of Clinical Studies of Drugs and Medical Devices of the Valencian Community in Spain.

\section{Eligibility criteria for the study}

For inclusion in the study, participants had to fulfill the following criteria: the volunteers were women aged 41-49 years old of all skin types (normal, dry, or oily) who regularly attended a dermatology clinic and had a predominantly urban lifestyle. They had to demonstrate the ability to understand the goal of the study, as well as the ability to understand any risks associated with their participation. Finally, they had to 
be fully capable of understanding and signing the informed consent document.

Women who had been smokers within the last 5 years, or who were regularly exposed to the sun without protection, or who used ultraviolet A tanning were not considered for the study. Women suffering from severe skin disorders, such as cutaneous carcinoma, melanoma, collagenosis (eg, systemic lupus, scleroderma), severe acne or rosacea, scars, and consequences of other skin diseases were also excluded. Other environmental and lifestyle factors that clinicians considered to significantly contribute to perceived age were taken into account. Lastly, aesthetic facial treatments, medical or surgical, were also criteria for exclusion.

\section{Data collection}

A set of parameters known as phenotypic features were defined to develop the algorithm. Data were collected using two measuring methods: 1) biophysical assessment by ANTERA 3D ${ }^{\circledR}$ device (Miravex Limited, Dublin, Ireland) ${ }^{20}$ and 2) clinical assessment by an expert panel.

\section{Data derived from the ANTERA 3D ${ }^{\circledR}$ device}

Biophysical parameters derived from using the ANTERA $3 \mathrm{D}^{\circledR}$ device were wrinkles and roughness, number of wrinkles, depth of wrinkles, length of wrinkles, skin pigmentation, concentration of melanin, distribution (heterogeneity) of melanin, superficial vascular component, concentration of hemoglobin, distribution (heterogeneity) of hemoglobin, facial furrows analysis, nasogenian furrow analysis, and roughness. Clinically assessed parameters were the position of eyebrows, state of periorbital wrinkles, state of facial wrinkles, and evaluation of the labiomental groove.

The phenotypes of all subjects involved in the study are represented by 22 variables that are shown in Table 1 .

\section{Data collected by the clinical assessment of an expert panel}

Data on the perceived age of the subjects measured using the test of Carruthers and Carruthers was collected using photographic images, which is the predominant method for generating perceived age. ${ }^{3}$

The photos were taken in a standardized manner, with reproducibility of lighting conditions, photographic settings, and other conditions like no makeup, covered hair, no jewelry. Subsequently, these photographs were assessed blindly and independently by a panel of experts consisting of six dermatologists, who assigned an age to each one of the
Table I Variables used to describe the phenotypic features of the skin

\begin{tabular}{l} 
Variables \\
\hline Position of eyebrows \\
State of periorbital wrinkles \\
Right periorbital zone \\
Left periorbital zone \\
State of facial wrinkles \\
Right frontal zone \\
Left frontal zone \\
Evaluation of labiomental groove \\
Wrinkles and roughness \\
Length of wrinkles \\
Thickness of wrinkles \\
Depth of wrinkles \\
Pigmentation \\
Average value of melanin \\
Variation-heterogeneity index of the melanin distribution \\
Relative percentage variation \\
Method of distribution of melanin in the study area \\
Superficial vascular component \\
Average value of hemoglobin \\
Variation-heterogeneity index of the hemoglobin distribution \\
Relative percentage variation \\
Method of distribution of hemoglobin in the study area \\
Depth and length of the facial nasogenian groove \\
Depth and length of the labiomental groove \\
Roughness \\
\hline
\end{tabular}

subjects on the basis of their expert opinion and professional experience as well as using the validated scale age of facial age gradation suggested by Carruthers and Carruthers. The Carruthers grading scale defines perceived age as an integer in the range of -5 to +5 years. For example, +2 indicates that a subject appears to be 2 years older than his or her actual age, whereas -3 indicates that the subject's perceived age is 3 years younger than his or her chronological age.

\section{Algorithm for diagnosing perceived age}

We developed an algorithm that simulates and improves the clinical assessment performed by the expert panel, determining a person's perceived age with the Carruthers validated facial grading scale based on individual phenotype criteria.

The algorithm is based on artificial intelligence procedures, specifically ANN. The system developed here employs supervised mathematical learning and error retropropagation techniques to learn the relations between biophysical and clinically assessed parameters of subjects. The algorithm takes phenotype data as variables and expert assignments as objective data that are used in the learning process. As a result, our method generates an improved simulation of expert assessments of perceived age for a given set of variables. 
The artificial neural network (ANNs) are trained by using the standard k-fold cross-validation method. In our case, we did this process ten times $(\mathrm{k}=$ ten $)$, so the original sample was randomly partitioned into ten equal-sized subsamples. Of the ten subsamples, a single subsample was retained as the validation data for testing the model, and the remaining nine subsamples were used as training data. The cross-validation process is then repeated ten times (the folds), with each of the ten subsamples used exactly once as the validation data. The ten results from the folds were then averaged to produce a single estimation of accuracy. The advantage of this method is that all observations are used for both training and validation, and each observation is used for validation exactly once. The averaged accuracy of the method is calculated by combining the results of the ten different training/validation data samples.

ANNs are capable of pattern recognition in complex data collections, as in our case. Supervised learning (or optimization) methods are directed specifically to infer patterns from large collections of data, in order to develop models that predict the correspondence between a set of variables and a set of objective data (in our case, the variables are the phenotypic evaluations, and objective data are the perceived ages).

In our case, the phenotypic evaluation for each subject is composed of 22 properties, ie, 22 different values that can be represented as a vector with 22 dimensions. In order to apply the ANN methods we needed to reduce the 22 dimensions to two, while preserving the original properties of the 22 original dimensions. A number of mathematical methods exist to perform this transformation; one of the most common techniques is Sammon mapping and back propagation. ${ }^{21}$

\section{Results and discussion}

A new and standardized method for diagnosing perceived age has been developed to replicate the clinical assessment performed by an expert panel. The method learns from the combination of two sets of data: phenotypic, objective and quantifiable variables, and expert assignments by qualified dermatologists.

We selected 22 variables as main contributors to perceived age estimation (Table 2). For example, our results indicate that the length of the nasogenian groove (11.67\%) contributes to perceived age in a much more significant manner than depth of wrinkles $(1.61 \%)$ or melanin concentration $(0.10 \%)$.

Considering the individual relevance of each variable in accordance with these values, we have developed an algorithm able to make an estimation of perceived age. The
Table 2 Effect of the contribution of each of 22 variables involved in the study

\begin{tabular}{ll}
\hline Variables & Relevance \\
\hline Length of nasogenian groove & $11.67 \%$ \\
Variation-heterogeneity index of the hemoglobin & $10.41 \%$ \\
distribution & \\
Depth of nasogenian groove & $10.26 \%$ \\
Position of eyebrows & $7.52 \%$ \\
Method of distribution of hemoglobin in the study area & $6.83 \%$ \\
Hemoglobin concentration & $6.70 \%$ \\
Method of distribution of melanin in the study area & $6.35 \%$ \\
Roughness & $6.28 \%$ \\
Variation-heterogeneity index of the melanin distribution & $6.25 \%$ \\
Percentage of melanin distribution & $4.56 \%$ \\
Length of wrinkles & $4.06 \%$ \\
Depth of labiomental groove & $3.71 \%$ \\
Thickness of wrinkles & $3.67 \%$ \\
Left frontal facial wrinkles & $2.63 \%$ \\
Length of labiomental groove & $2.35 \%$ \\
Depth of wrinkles & $1.61 \%$ \\
Right frontal facial wrinkles & $1.26 \%$ \\
Percentage of hemoglobin distribution & $1.25 \%$ \\
Left periorbital wrinkles & $0.81 \%$ \\
Right periorbital wrinkles & $0.33 \%$ \\
Melanin concentration & $0.10 \%$ \\
\hline
\end{tabular}

algorithm was able to correctly classify the samples with an accuracy of $92.04 \%$, where the accuracy is the capability to predict the perceived age estimated by the experts, having as input variables the 22 selected ones. Therefore, we have demonstrated that the suggested method is valid for determining perceived age of a subject in a standardized, consistent manner.

\section{Conclusion}

There is an urgent need for objective, quantitative, and standardized rating methods capable of performing an accurate diagnosis of perceived age. In this paper, we propose a novel artificial intelligence-based method that successfully emulates and improves the clinical assessment performed by an expert panel. With an accuracy of $92 \%$, it can represent a valid substitute for traditional approaches, which are costly, time-consuming, and potentially unreliable.

Expert assessments rely on human subjective assignments exposed to individual bias. That is, there may be differences in judgment between experts, and also the criteria of one individual might change with time. Furthermore, the recruitment of a panel of dermatologists is time-consuming and entails considerable cost. In comparison, a computerized method is free from these shortcomings.

The ability to quantify the aesthetic signs of aging on the basis of a given set of parameters is highly appealing for the 
cosmetics industry. Potential applications include the design of a personalized treatment for an individual depending on his or her apparent age, or keeping track of the response to anti-aging treatment. Also, it could be used as a validation criterion for treatments comprising cosmetics, aesthetic surgery, alternative medicine, exercise, or diets. Going even further, as there is a clear relationship between perceived age and biological age, our method could be employed to evaluate the overall health status of an individual ${ }^{16}$ in an age group (41-49 years old) where the most cost-effective interventions (therapeutic or of lifestyle changes) can be applied.

\section{Disclosure}

The authors report no conflicts of interest in this work.

\section{References}

1. PRWeb. Global cosmetics manufacturing industry market research report now available from IBISWorld [homepage on the Internet]. Los Angeles, CA: Vocus PRW Holdings, LLC; 2012. Available from: http://www.prweb.com/releases/2012/9/prweb9905942.html. Accessed September 19, 2013.

2. Karasik D, Demissie S, Cupples LA, Kiel DP. Disentangling the genetic determinants of human aging: biological age as an alternative to the use of survival measures. J Gerontol A Biol Sci Med Sci. 2005;60(5): 574-587.

3. Gunn DA, Rexbye H, Griffiths CE, et al. Why some women look young for their age. PLoS One. 2009;4(12):e8021.

4. Noordam R, Gunn DA, Tomlin CC, et al; Leiden Longevity Study Group. High serum glucose levels are associated with a higher perceived age. Age (Dordr). 2013;35(1):189-195.

5. Noordam R, Gunn DA, Tomlin CC, et al; Leiden Longevity Study group. Cortisol serum levels in familial longevity and perceived age: the Leiden longevity study. Psychoneuroendocrinology. 2012;37(10): 1669-1675.

6. Rexbye H, Petersen I, Johansens M, Klitkou L, Jeune B, Christensen K. Influence of environmental factors on facial ageing. Age Ageing. 2006;35(2):110-115.

7. Tafet GE, Idoyaga-Vargas VP, Abulafia DP, et al. Correlation between cortisol level and serotonin uptake in patients with chronic stress and depression. Cogn Affect Behav Neurosci. 2001;1(4):388-393.
8. Farage MA, Miller KW, Elsner P, Maibach HI. Intrinsic and extrinsic factors in skin ageing: a review. Int J Cosmet Sci. 2008;30(2):87-95.

9. Matts PJ, Fink B, Grammer K, Burquest M. Color homogeneity and visual perception of age, health, and attractiveness of female facial skin. J Am Acad Dermatol. 2007;57(6):977-984.

10. Fink B, Matts PJ, D’Emiliano D, Bunse L, Weege B, Röder S. Colour homogeneity and visual perception of age, health and attractiveness of male facial skin. J Eur Acad Dermatol Venereol. 2012;26(12):1486-1492.

11. Ozdemir R, Kilinç H, Unlü RE, Uysal AC, Sensöz O, Baran CN. Anatomicohistologic study of the retaining ligaments of the face and use in face lift: retaining ligament correction and SMAS plication. Plast Reconstr Surg. 2002;110(4):1134-1147; discussion 1148-1149.

12. Taub AF, Sarnoff D, Gold M, Jacob C. Effect of multisyringe hyaluronic acid facial rejuvenation on perceived age. Dermatol Surg. 2010;36(3):322-328.

13. Chauhan N, Warner JP, Adamson PA. Perceived age change after aesthetic facial surgical procedures quantifying outcomes of aging face surgery. Arch Facial Plast Surg. 2012;14(4):258-262.

14. Guinot C, Malvy DJ, Ambroisine L, et al. Relative contribution of intrinsic vs extrinsic factors to skin aging as determined by a validated skin age score. Arch Dermatol. 2002;138(11):1454-1460.

15. Bulpitt CJ, Markowe HL, Shipley MJ. Why do some people look older than they should? Postgrad Med J. 2001;77(911):578-581.

16. Carruthers A, Carruthers J. A validated facial grading scale: the future of facial ageing measurement tools? $J$ Cosmet Laser Ther. 2010;12(5):235-241.

17. Oizumi T, Daimon M, Wada K, et al. A proposal for the cutoff point of waist circumference for the diagnosis of metabolic syndrome in the Japanese population. Circ J. 2006;70(12):1663.

18. de Koning L, Merchant AT, Pogue J, Anand SS. Waist circumference and waist-to-hip ratio as predictors of cardiovascular events: meta-regression analysis of prospective studies. Eur Heart $J$. 2007;28(7):850-856.

19. Sparavigna A, Guglielmini G, Togni S, Cristoni A, Maramaldi G. Evaluation of anti-cellulite efficacy: a topical cosmetic treatment for cellulite blemishes - a multifunctional formulation. J Cosmet Sci. 2011;62(3):305-316.

20. Miravex. Antera $3 \mathrm{D}^{\circledR}$, analysis of your skin [homepage on the Internet]. Dublin, Ireland: Miravex Limited; 2013. Available from: http://miravex. com/english/antera_3d/antera_3d_analysis_of_your_skin-4.html. Accessed September 19, 2013.

21. J. Sun, C. Fyfe, M. Crowe. Extending Sammon mapping with Bregman divergences. Information Sciences. 2012;187:72-92.
Clinical, Cosmetic and Investigational Dermatology

\section{Publish your work in this journal}

Clinical, Cosmetic and Investigational Dermatology is an international, peer-reviewed, open access, online journal that focuses on the latest clinical and experimental research in all aspects of skin disease and cosmetic interventions. All areas of dermatology will be covered; contributions will be welcomed from all clinicians and

\section{Dovepress}

basic science researchers globally. This journal is indexed on CAS. The manuscript management system is completely online and includes a very quick and fair peer-review system, which is all easy to use. Visit http://www.dovepress.com/testimonials.php to read real quotes from published authors. 